\title{
Research of Service Restoration Reconfiguration in Distribution Network Junfeng Wang ${ }^{1, a}$, Hongming Zhou ${ }^{2, b}$
}

${ }^{1}$ School of Physics and Electronic Engineering of Yuxi Normal University, Yuxi, 653100, China

${ }^{2}$ School of Physics and Electronic Engineering of Yuxi Normal University, Yuxi, 653100, China

awjf@yxnu.net, bzhouhm@yxnu.net

Keywords: Distribution Network; Service Restoration; Algorithms for Reconfiguration

\begin{abstract}
The purpose of this paper is to propose a mixed integer programming model of service restoration reconfiguration considering the minimum switching frequency and power losses. The recovery after the service failure of distribution network is a multi-objective optimization problem with constraints. This paper also discusses the reconfiguration algorithms for power restoration and load balancing in the real-time operation environment of distribution network, which can make the problem solving easy to handle and control. The methods and measures for service auto restoration in distribution automation system are analyzed by examples.
\end{abstract}

\section{Introduction}

Distribution system reconfiguration is the topological structure which changes network by changing the switch state in the normal or abnormal operating conditions ${ }^{[1]}$. The main function of the service restoration reconfiguration program is to help dispatchers to make decisions in power restoration procedure, and its goal is transferring the load of the fault section into normal feeders as much as possible by network reconfiguration with permissible operating conditions and electrical constraint. Thus, the service restoration reconfiguration is mainly to restore power for non-fault power outage section below the fault section.

Most of the algorithms for service restoration reconfiguration nowadays is based on the switching heuristic searching algorithm ${ }^{[2-4]}$. The major advantage of these algorithms is fast. Since each switching operation needs more time and manpower, many service restoration reconfiguration algorithms aim to minimize switching frequency ${ }^{[5}$. In order to obtain the efficient and robust performance, this paper employs the service restoration reconfiguration model in distribution network in the minimum of switching frequency and power losses, which can provide operating crew with supporting tools for real-time operation decisions.

\section{Mathematical model}

\subsection{Definition}

Node switch: A switch disconnected normally which connects two adjacent feeders.

Fault section: A part of the feeder with lasting breakdown.

Non-fault power outage section: A part of the feeder in the downstream of the fault section, which can be separate from the fault section by disconnecting a closed switch.

Adjacent feeder: A feeder connecting the node switch to the feeder which is in the fault state or overloaded.

Second-level feeder: A feeder which is connected to the adjacent feeder with the node switch, while with no node switch to the feeder in the fault state or overloaded.

Boundary switch: A switch disconnected normally which connects the power outage section(or section with overload line) with the adjacent feeder(or a non-fault line of the feeder in the fault state or overloaded).

Equivalent load: The total amount of all the load in the track section connected by switches(closed or open). 


\subsection{Objective function}

\subsubsection{Service restoration in the minimum of switching frequency}

The objective function can be expressed as:

$$
\begin{aligned}
& \min =\left\{\sum_{i=1}^{m}\left(1-y_{i}\right)+\sum_{j=1}^{n} z_{j}\right\} \\
& y_{i}=\left\{\begin{array}{ll}
1, & \text { switch i remains closed in the reconfiguration } \\
0, & \text { switch } \mathrm{i} \text { is turned on in the reconfiguration }
\end{array} \quad i=1,2, \ldots, m\right. \\
& z_{j}=\left\{\begin{array}{ll}
1, & \text { boundary switch } \mathrm{j} \text { is closed in the reconfiguration } \\
0, & \text { boundary switch } \mathrm{j} \text { remains open in the reconfiguration }
\end{array} \quad j=1,2, \ldots, n\right.
\end{aligned}
$$

Where $Y=\left[y_{1}, y_{2}, \ldots, y_{m}\right]^{T}$ represents the change of closed switches of non-fault power outage section before breakdown in the reconfiguration; $Z=\left[z_{1}, z_{2}, \ldots, z_{n}\right]^{T}$ represents the change of open interconnection switches which is connected with non-fault power outage section in the reconfiguration.

\subsubsection{Comprehensive service restoration model}

In general, the service restoration gives priority to the power restoration of all the load in the minimum of switching frequency. In the actual distribution network, some switches can be controlled remotely, which should be chosen first. Considering all above, we propose a comprehensive service restoration objective function:

$$
\min =\left\{\sum_{i=1}^{m} a_{i}\left(1-y_{i}\right)+\sum_{j=1}^{n}\left(a_{m}+j z_{j}\right)+\sum_{i=1}^{n_{2}} I_{i}^{2} R_{i}\right\}
$$

Where $A=\left[a_{1}, \ldots, a_{m}, a_{m+1}, \ldots, a_{m+n}\right]$ is a weight vector. When $a_{i}$ is large, the objective function should consider the minimum switching frequency first, then the minimum power losses. $a_{i}$ can take the same value in general, namely, the weight of each switch is the same; when taking remote control switches into account, the value of $a_{i}$ corresponding to each switch could be smaller. In other words, if choosing remote control switches, a little higher switching frequency is allowed. $I=\left[I_{1}, I_{2}, \ldots, I_{n_{2}}\right]$ is the current amplitude of each branch in distribution system; $R_{i}$ is the resistance of each branch; and $n_{2}$ is the amount of the whole distribution system branches, which can only choose branches in the feeder related to the service restoration in the practical computation $^{[6]}$.

\subsection{Constraint condition}

\subsubsection{Description of topological constraint}

Connectivity constraint is that each load has at least one power supply point, namely, closed interconnection switches must be more than open disconnecting switches in service restoration, otherwise there will be some isolated nodes, which can be expressed as:

$$
\left(1-y_{i}\right)+\sum_{k \in \Omega_{i}}\left(1-y_{k}\right)-\sum_{l \in \Psi_{i}} z_{l} \leq 0
$$

Where $i=1,2, \ldots, m ; \Omega_{i}$ is the set of closed switches below the closed switch i(with respect to the power); $\Psi_{i}$ is the set of boundary switches below the closed switch i(with respect to the power).

\subsubsection{Constraint on distribution system radiation}

Radiation constraint is that each load has only one power supply point and looped network cannot appear, namely, at least one of the branches connecting two closed interconnection switches should be disconnected , which can be expressed as:

$$
\sum_{k \in \text { loop }_{i j}}\left(1-y_{k}\right)-\left(z_{i}+z_{j}\right)+1 \geq 0
$$

Where loop $_{i j}$ is the set of closed switches between switch i and switch $\mathrm{j}$. 


\subsubsection{Capacity constraint of boundary switch}

The total load allocated to the boundary switch should be less than its capacity margin, which is

$$
\sum_{j=1}^{m_{2}} x_{i j} S_{j} \leq M_{k} \quad i=1,2, \cdots, n
$$

Where $m_{2}$ is the amount of load in non-fault power outage section; $k \in \Psi_{i}, \Psi_{i}$ is the set of branches from the boundary switch $\mathrm{i}$ along the line to the power supply point; $M_{k}$ is capacity margin of the boundary switch $\mathrm{k}$.

\section{Example application}

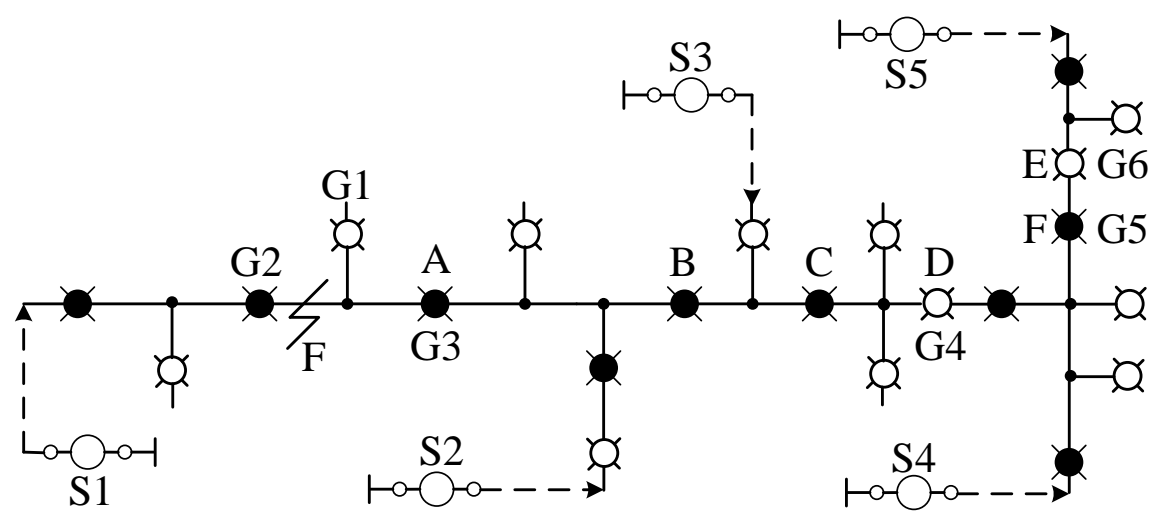

Fig.1. The power supply diagram in one district

Let's take figure 1 as example, after closing the power transmission switch S1 in distribution network, power supply between the power S1 and the knife switch G2 will be restored, while the non-fault side of the knife switch G3 will not be restored. Then we find the trunk circuit and close the open knife switch G4 which is the farthest from the trunk circuit, and put it as the isolation point. Thus the trunk circuit is divided into three main line sections: $A B, B C$, and $C D$, then we search respectively whether or not they have backup power. There is a backup power S3 in the line section $\mathrm{BC}$, and we calculate the minimum value of its redundant capacity, which is the redundant capacity minima of primary equipment, cable, and circuit of each level; if the value is bigger than the load in the line section AD, we should close the power switch and restore the power supply of its users. Since the power S3 supplies power to both AC and BD, the power outage line section AC, which is close to the fault point, should be considered first, and BC should be given priority to power supply by S3. As for other line sections, we should first consider their power redundant capacity, for example, AD can be powered by S2 after calculation, while CD cannot be powered by the closest power S4 because the redundant capacity of S4 is insufficient, so we can transfer some of the load in S4 into other power.

The backup power should be considered first when transferring, then other branch power. Some of the load in S4 can be transferred into S5 after calculation, which is closing the knife switch G6 and disconnecting G5. When the line load in S4 cannot be transferred, we should find whether there are other branches in CD whose redundant capacity is bigger than zero and if so, we will transfer the load, and if not, we will inform dispatchers of disposing the switched capacitor, regulating the tap position of the on-load voltage regulator, etc.

\section{Conclusion}

This paper aims to study the reconfiguration problem after service failure of distribution network, and propose a mixed integer programming model of service restoration reconfiguration at the minimum of switching frequency and power losses. The searching algorithm is fit for solving integer programming problems and mixed integer programming problems, thus the given model of service restoration can be solved efficiently by using the algorithm. 


\section{References}

[1] Xiufan Le, Chengfeng Yang, Qingshan Xu. Research of the reconfiguration algorithm of service restoration and load equalization in distribution network[J]. Power System Technology, 2002, 26(7): 34-37.

[2] Wu J S, Tonsovic K L, Chen C S. A heuristic search approach to feeder switching operation for overload, faults, unbalance flow and maintenance[J]. IEEE Trans on Power Delivery, 1991, 6(4).

[3] Hsu Yuan-Yih, Kuo H. A heuristic based fuzzy reazoning approach for distribution system service restoration[J]. IEEE Trans on Power Delivery, 1994, 9(4): 1950-1957.

[4] Miu Karen Nan, Chiang Hsiao- Dong, Yuan Bentao, et al. Fast Service Restoration for Large-Scale Distribution Systems with Priority Customers and Constraints[J]. IEEE Trans on Power Systems, 1998, 13(3).

[5] Shirmohammadi D. Service restoration in distribution networks via network reconfigration[J]. IEEE Transaction on Power Delivery, 1992, 7(4): 952-958.

[6] Haifeng Li, Yao Zhang. Research of the algorithm for service restoration reconfiguration in distribution network[J]. Power System Automation, 2001, 4: 34-37. 